\title{
Genericity under parahoric restriction
}

\author{
Manish Mishra* $\quad$ Mirko Rösner ${ }^{\dagger}$
}

\begin{abstract}
We study the preservation of genericity under parahoric restriction of depth zero representations.
\end{abstract}

\section{Introduction}

Let $\mathbf{G}$ be a connected reductive group defined over a non-archimedean local field $k$. Let $\mathbf{B}$ be a $k$-Borel subgroup of $\mathbf{G}$ with unipotent radical $\mathbf{U}$ and let $\mathbf{T}$ be a maximal $k$-torus in $\mathbf{B}$. The corresponding groups of $k$-rational points are $G, B, U, T$. A character $\psi: U \rightarrow \mathbb{C}^{\times}$ is called generic if the stabilizer of $\psi$ in $T$ is exactly the center $Z$ of $G$. An admissible representation $\pi$ of $G$ is called generic (more specifically $\psi$-generic) if there exists a generic character $\psi$ of $U$ such that $\operatorname{Hom}_{G}\left(\pi, \operatorname{Ind}_{U}^{G} \psi\right) \neq 0$.

A basic result due to Rodier [4, Thm. 2] states that genericity is preserved under the Jacquet functor. On the category of depth zero representations of $G$ (see [2] for the notion of depth), there is a functor analogous to the Jacquet functor called the parahoric restriction functor [7]. It is defined as follows. Let $G_{x}$ be a parahoric subgroup of $G$ with prounipotent radical $G_{x}^{+}$. The quotient $G_{x} / G_{x}^{+}$is the $\mathbb{F}_{q}$-points of a connected reductive group $\underline{\mathbf{M}}$ defined over $\mathbb{F}_{q}$, where $\mathbb{F}_{q}$ is the residue field of $k$. The parahoric restriction functor sends a representation $(\pi, V)$ of $G$ to the representation of $\underline{\mathbf{M}}\left(\mathbb{F}_{q}\right)$ obtained by restricting $\pi$ to $G_{x}$ and then taking the $G_{x}^{+}$-invariants of it.

We say $\mathbf{G}$ is unramified, if it is quasisplit and splits over an unramified extension. Suppose that $\mathbf{G}$ is unramified, that $G_{x}$ is contained in a hyperspecial parahoric subgroup of $G$ and that $\pi$ is parabolically induced from a supercuspidal representation of a Levi subgroup of

\footnotetext{
${ }^{*}$ University of Heidelberg, Germany, electronic address: manish.mishra@gmail.com

${ }^{\dagger}$ University of Heidelberg, Germany, electronic address: mirko_rosner@hotmail.com
} 
$G$. Then we show in Theorem 2 that the parahoric restriction functor preserves genericity of $\pi$. This does not generalize to arbitrary parahorics and admissible representations, see Section 5 .

\section{Notations}

Fix a non-archimedean local field $k$ and an unramified connected reductive group $\mathbf{G}$ over $k$. Let $\mathbf{B}=\mathbf{T} \ltimes \mathbf{U}$ be a $k$-Borel subgroup of $\mathbf{G}$ with a maximal $k$-torus $\mathbf{T}$ and a unipotent radical $\mathbf{U}$. Let $\mathbf{Z}$ be the center of $\mathbf{G}$ and let $\mathbf{T}_{\mathrm{ad}}:=\mathbf{T} / \mathbf{Z}$. Their groups of $k$-rational points are $G, B, T, U, Z$ and $T_{\text {ad }}$, respectively. We will follow the standard abuse of notation to write "parabolic subgroups of $G$ " instead of " $k$-points of $k$-parabolic subgroups of G".

For a point $x$ in the Bruhat-Tits building of $G$, the associated parahoric subgroup will be denoted by $G_{x}$. Its Levi quotient is $\underline{G_{x}}=G_{x} / G_{x}^{+}$with the pro-unipotent radical $G_{x}^{+}$. We will denote by $\operatorname{Rep}(G)$ the category of admissible complex representations of $G$ and likewise for the other groups.

\section{Parahoric restriction functor}

Fix a point $x$ in the apartment attached to $T$. Restricting an admissible representation $(\pi, V)$ of $G$ to the parahoric $G_{x}$ and taking invariants with respect to the pro-unipotent radical $G_{x}^{+}$gives rise to a representation of $\underline{G_{x}}=G_{x} / G_{x}^{+}$. This defines the parahoric restriction functor

$$
\mathbf{r}_{G_{x}}: \operatorname{Rep}(G) \rightarrow \operatorname{Rep}\left(\underline{G_{x}}\right), \quad\left\{\begin{array}{l}
(\pi, V) \longmapsto\left(\underline{G_{x}} \rightarrow \operatorname{Aut}\left(V^{G_{x}^{+}}\right)\right), \\
\left(V_{1} \rightarrow V_{2}\right) \longmapsto\left(V_{1}^{G_{x}^{+}} \rightarrow V_{2}^{G_{x}^{+}}\right)
\end{array}\right.
$$

where $V_{1} \rightarrow V_{2}$ is a morphism between admissible representations $\left(\pi_{1}, V_{1}\right)$ and $\left(\pi_{2}, V_{2}\right)$. This functor is exact and defines a homomorphism between the corresponding Grothendieck groups, analogous to Jacquet's functor of parabolic restriction.

For hyperspecial parahorics, parahoric restriction commutes with parabolic induction in the following sense:

Theorem 1. Let $x$ be a hyperspecial point in the apartment attached to $T$ with corresponding hyperspecial parahoric subgroup $G_{x} \subseteq G$. Fix a standard parabolic subgroup $P \supseteq B$ with Levi decomposition $P=M \ltimes N$ such that $T \subseteq M$. Then the following diagram is commutative 
up to natural equivalence:

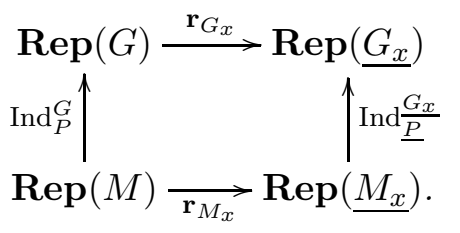

The parabolic subgroup $\underline{P}=\left(G_{x} \cap P\right) /\left(G_{x}^{+} \cap P\right)$ of $\underline{G_{x}}$ is generated by the same roots as $P$ and has Levi subgroup $\underline{M_{x}}$.

Proof. The parahoric subgroup of $M$ attached to $x$ is $M_{x} \cong G_{x} \cap M$, cp. [2].

Fix an admissible representation $(\sigma, V)$ of $M$ and denote its inflation to $P$ by the same symbol. We construct a natural equivalence $\left(\operatorname{Ind}_{P}^{G}(\sigma)\right)^{G_{x}^{+}} \rightarrow \operatorname{Ind} \underline{\underline{G_{x}}}\left(\mathbf{r}_{M_{x}}(\sigma)\right)$. $\operatorname{Then}_{\operatorname{Ind}}^{G}(\sigma)$ has a canonical model by right multiplication on the space of functions $f: G \rightarrow V$ with

$$
f(p g)=\delta_{P}^{1 / 2}(p) \sigma(p) f(g) \quad \forall p \in P, g \in G,
$$

where $\delta_{P}$ is the modulus character. By the Iwasawa decomposition $G=P G_{x}$, every such $f$ is uniquely defined by its restriction $\tilde{f}=\left.f\right|_{G_{x}}$ to $G_{x}$. The linear map $f \mapsto \tilde{f}$ is thus a $G_{x^{-}}$equivariant isomorphism from $\operatorname{Ind}_{P}^{G}(\sigma)$ to the space of $\tilde{f}: G_{x} \rightarrow V$ with

$$
\tilde{f}(p g)=\sigma(p) \tilde{f}(g) \quad \forall p \in G_{x} \cap P, g \in G_{x}
$$

Such an $\tilde{f}: G_{x} \rightarrow V$ is invariant under the right action of $G_{x}^{+}$if and only if $\tilde{f}(x u)=\tilde{f}(x)$ for every $u \in G_{x}^{+}$and $x \in G_{x}$. In that case we have

$$
\sigma(p) \tilde{f}(g)=\tilde{f}(p g)=\tilde{f}\left(g g^{-1} p g\right)=\tilde{f}(g) \quad \forall p \in P \cap G_{x}^{+}, \quad g \in G_{x},
$$

since $g^{-1} p g \in G_{x}^{+}$. Hence $\tilde{f}$ actually maps into the invariant space $V^{P \cap G_{x}^{+}}$.

Now $\tilde{f}$ factors over a unique function $h_{f}: \underline{G_{x}} \rightarrow V^{P \cap G_{x}^{+}}$with the property

$$
h_{f}(\underline{p g})=\mathbf{r}_{M_{x}}(\sigma)(\underline{p}) h_{f}(\underline{g}) \quad \forall \underline{p} \in \underline{P} \underline{g} \in \underline{G} .
$$

The action of $\underline{G_{x}}$ by right-multiplication on the space of these $h_{f}$ is a model of the induced representation $\operatorname{Ind}_{\underline{P}} \circ \mathbf{r}_{M_{x}}(\sigma)$ of $\underline{G_{x}}$. The family of isomorphisms $\left\{f \mapsto h_{f}\right\}_{\sigma}$ provides the natural equivalence. 


\section{Generic depth zero}

A character $\psi: U \rightarrow \mathbb{C}^{\times}$is called generic if its stabilizer in $T_{\text {ad }}$ is trivial. Let $z$ be a hyperspecial vertex of $G$ in the apartment attached to $T$. A generic character $\psi$ of $U$ is called depth-zero at $z$ if its restriction to $U \cap G_{z}$ factors through a generic character $\psi_{z}$ of $\underline{U}:=\left(U \cap G_{z}\right) /\left(U \cap G_{z}^{+}\right)$.

Theorem 2. Let $\pi=\operatorname{Ind}_{P, M}^{G}(\sigma)$ be an irreducible admissible representation of $G$ that is parabolically induced from a supercuspidal irreducible representation $\sigma$ of a Levi subgroup $M$ of a parabolic $P \subseteq G$. Let $G_{x}$ be a parahoric subgroup of $G$, contained in a hyperspecial parahoric subgroup, such that $\underline{\pi}:=\mathbf{r}_{G_{x}}(\pi) \neq 0$. Then $\pi$ being generic implies that $\underline{\pi}$ is generic.

Proof. By conjugating $x$ and $P$ if necessary, we can assume without loss of generality that $P \supseteq B$, that $M \supseteq T$, that $x$ is a point in the apartment associated to $T$ and that $\pi$ is generic with respect to a generic character $\psi$ of $U$. We can assume without loss that $G_{x}$ is a hyperspecial maximal parahoric subgroup of $G$ because of transitivity of parahoric restriction [7, 4.1.3] and Rodier's result [4, Thm. 2].

If $w_{o}$ is an element in the normalizer of $T$ such that $B \cap w_{o} B w_{o}^{-1}=T$, then $Q:=$ $M \cap w_{o} U w_{o}^{-1}$ is a maximal unipotent subgroup of $M[4, \mathrm{Thm} .2]$. Define a generic character of $Q$ by $\psi_{M}(q)=\psi\left(w_{o}^{-1} q w_{o}\right)$ for $q \in Q$. Then by [4, Thm. 2],

$$
\operatorname{Hom}_{U}(\pi, \psi) \cong \operatorname{Hom}_{Q}\left(\sigma, \psi_{M}\right)
$$

Since $\underline{\pi} \neq 0$, Theorem 1 implies that $\sigma$ is a depth zero supercuspidal representation of $M$. By [1, Lemma 6.1.2], there is a hyperspecial point $y$ of $M$ and a cuspidal representation $\tau^{\circ}$ of $\underline{M_{y}}$ such that

a) $\psi_{M}$ is depth zero at $y$ and $\tau^{\circ}$ is $\left(\psi_{M}\right)_{y}$-generic.

b) There is an extension of $\tau^{\circ}$ to a representation $\tau$ of the normalizer $\left[M_{y}\right]$ of $M_{y}$ in $M$ such that $\sigma=\mathrm{c}-\operatorname{Ind}_{\left[M_{y}\right]}^{M} \tau$. Note that since $y$ is a hyperspecial point of $M,\left[M_{y}\right]=$ $Z_{M} M_{y}$, where $Z_{M}$ denotes the center of $M$.

Since $\sigma$ has depth zero at $x$ by Thm. 1, we can assume without loss of generality that $x=y$ (see proof of [8, Lemma 3.3(ii)]). We have therefore

$$
\operatorname{Hom}_{\underline{Q}}\left(\tau^{\circ},\left(\psi_{M}\right)_{x}\right) \neq 0
$$


where $Q$ denotes a maximal unipotent subgroup of $M_{x}$ defined in the same way as $Q$.

Theorem 1 and a result of Vignéras [6, §7] imply that $\underline{\pi}$ is isomorphic to $\operatorname{Ind} \frac{G_{x}}{\underline{P}} \tau^{\circ}$. Then again by [4, Thm.2],

$$
\operatorname{Hom}_{\underline{U}}\left(\underline{\pi}, \psi_{x}\right) \cong \operatorname{Hom}_{\underline{Q}}\left(\tau^{\circ},\left(\psi_{M}\right)_{x}\right) \neq 0 .
$$

This completes the proof.

\section{Non-special parahoric restriction}

In Theorem 2 we make two technical assumptions: we assume that $\pi$ is parabolically induced and that $G_{x}$ is contained in a hyperspecial maximal parahoric subgroup. However, if we drop these assumptions, there is a counterexample.

Counterexample: There is a generic irreducible admissible representation $\pi$ of $G=$ $\operatorname{GSp}(4, k)$ and a parahoric subgroup $G_{x} \subseteq G$ such that the parahoric restriction $\mathbf{r}_{G_{x}}(\pi)$ is non-zero, but not generic.

Proof. Let $\xi$ be the non-trivial unramified quadratic character of $k^{\times}$. Let $G=\operatorname{GSp}(4, k)$ be the group of symplectic similitudes with respect to the symplectic form $\left({ }_{-w} w\right)$ for $w=\left({ }_{1}{ }^{1}\right)$. Fix the Borel pair $(B, T)$ where $B=T \ltimes U \subseteq G$ is the subgroup of upper triangular matrices and $T \subseteq B$ is the maximal torus of diagonal matrices. Fix a character

$$
\alpha: T \rightarrow \mathbb{C}^{\times}, \quad \operatorname{diag}(a, b, c / a, c / b) \mapsto \xi(a b)|a||c|^{-1 / 2} .
$$

Inflating $\alpha$ to $B$ gives rise to the induced representation $\operatorname{Ind}_{B}^{G} \alpha$, which admits a unique irreducible subrepresentation ${ }^{1} \pi$. The representation $\pi$ is generic for arbitrary with respect to every generic character $\psi$ of $G$.

The standard paramodular subgroup $G_{x} \subseteq G$ is a non-special maximal parahoric subgroup. The second author has shown [5, Thm. 3.7] that the parahoric restriction $\mathbf{r}_{G_{x}}(\pi)$ is isomorphic to the restriction of $\mathbf{1} \otimes \mathrm{St} \oplus \mathrm{St} \otimes \mathbf{1}$ to

$$
\left\{\left(\xi_{1}, \xi_{2}\right) \in\left(\mathrm{GL}\left(2, \mathbb{F}_{q}\right)\right)^{2} \mid \operatorname{det} \xi_{1}=\operatorname{det} \xi_{2}\right\} \cong \underline{G_{x}} .
$$

where $\mathbf{1}$ is the trivial and St is the Steinberg representation of $\operatorname{GL}\left(2, \mathbb{F}_{q}\right)$. Thus $\mathbf{r}_{G_{x}}(\pi)$ is not generic.

\footnotetext{
${ }^{1}$ This is type Va in the notation of Roberts and Schmidt [3].
} 


\section{Acknowledgment}

The authors are thankful to Sandeep Varma for pointing out a mistake in the earlier draft of this article.

\section{References}

[1] S. DeBacker and M. Reeder. Depth-zero supercuspidal L-packets and their stability. Ann. of Math. (2), 169(3):795-901, 2009.

[2] A. Moy and G. Prasad. Jacquet functors and unrefined minimal K-types. Commentarii Mathematici Helvetici, 71(1):98-121, 1996.

[3] B. Roberts and R. Schmidt. Local Newforms for GSp(4), volume 1918 of Lecture Notes in Mathematics. Springer, 1 edition, October 2007.

[4] F. Rodier. Whittaker models for admissible representations of reductive $p$-adic split groups. In Harmonic analysis on homogeneous spaces (Proc. Sympos. Pure Math., Vol. XXVI, Williams Coll., Williamstown, Mass., 1972), pages 425-430. Amer. Math. Soc., Providence, R.I., 1973.

[5] M. Rösner. Parahoric restriction for GSp(4) and the inner cohomology of Siegel modular threefolds. PhD thesis, Ruprecht-Karls-Universität Heidelberg, 2016.

[6] M.-F. Vignéras. Irreducible Modular Representations of a reductive $p$-adic group and simple Modules for Hecke Algebras. In C. Casacuberta et al., editors, European Congress of Mathematics, Barcelona, volume 201 of Progress in Mathematics, pages 117-133. Birkhäuser, 2001.

[7] M.-F. Vignéras. Schur algebras of reductive p-adic groups, I. Duke Mathematical Journal, 116(1):35-75, 2003.

[8] J.-K. Yu. Construction of tame supercuspidal representations. J. Amer. Math. Soc., 14(3):579-622 (electronic), 2001. 